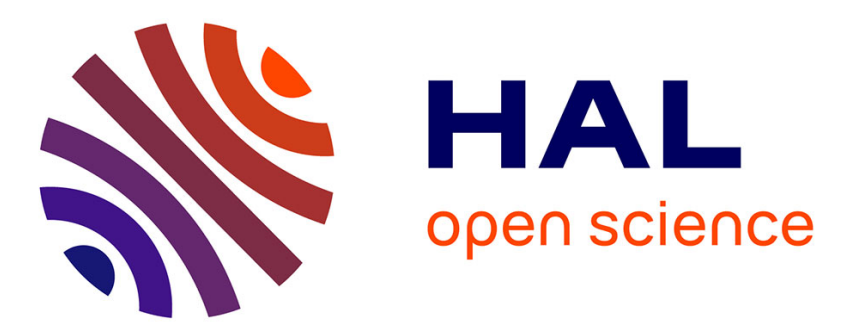

\title{
NMR relaxivity of coated and non-coated size-sorted maghemite nanoparticles
}

Jérôme Fresnais, Qianqian Ma, Linda Thai, Patrice Porion, Pierre Levitz, Anne-Laure Rollet

\section{- To cite this version:}

Jérôme Fresnais, Qianqian Ma, Linda Thai, Patrice Porion, Pierre Levitz, et al.. NMR relaxivity of coated and non-coated size-sorted maghemite nanoparticles. Molecular Physics, 2018, 117 (7-8), pp.990-999. 10.1080/00268976.2018.1527410 . hal-02173384

\section{HAL Id: hal-02173384 \\ https://hal.science/hal-02173384}

Submitted on 21 Nov 2019

HAL is a multi-disciplinary open access archive for the deposit and dissemination of scientific research documents, whether they are published or not. The documents may come from teaching and research institutions in France or abroad, or from public or private research centers.
L'archive ouverte pluridisciplinaire HAL, est destinée au dépôt et à la diffusion de documents scientifiques de niveau recherche, publiés ou non, émanant des établissements d'enseignement et de recherche français ou étrangers, des laboratoires publics ou privés. 


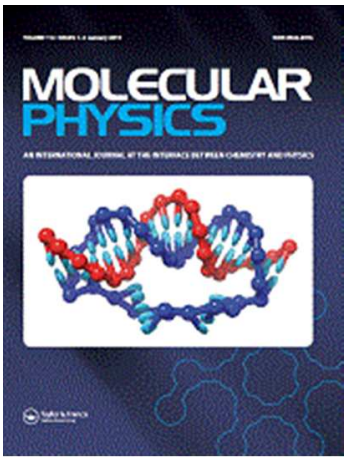

\section{NMR relaxivity on coated and non-coated size-sorted maghemite nanoparticles}

\begin{tabular}{|r|l|}
\hline Journal: & Molecular Physics \\
\hline Manuscript ID & TMPH-2018-0415 \\
\hline Danuscript Type: & Special Issue Paper \\
\hline Complete List of Authors: & $\begin{array}{l}\text { Fresnais, Jerome; Physicochimie des Electrolytes et Nanosystemes } \\
\text { Interfaciaux, } \\
\text { Ma, QIanQian; Physicochimie des Electrolytes et Nanosystemes } \\
\text { Interfaciaux } \\
\text { Thai, Linda; Physicochimie des Electrolytes et Nanosystemes Interfaciaux } \\
\text { Porion, Patrice; ICMN } \\
\text { Levitz, Pierre; Université Pierre et Marie Curie, Paris 6, Laboratoire PHENIX } \\
\text { Rollet, Anne-Laure; University Pierre et Marie Curie, }\end{array}$ \\
\hline Keywords: & maghemite nanoparticles, polymer coating, NMR relaxivity \\
\hline \multicolumn{2}{|c}{} \\
\hline
\end{tabular}

\section{SCHOLARONE}

Manuscripts 


\section{NMR relaxivity on coated and non-coated size-sorted maghemite nanoparticles}

Jérôme Fresnais ${ }^{1}{ }^{*}$, QianQian $\mathrm{Ma}^{l}$, Linda Thai ${ }^{1}$, Patrice Porion ${ }^{2}$, Pierre Levitz ${ }^{1}$ and Anne-Laure Rollet ${ }^{1}$ *

1 Sorbonne Université, CNRS, Laboratoire de Physico-chimie des Electrolytes et Nanosystèmes Interfaciaux, PHENIX - UMR 8234, F-75252 Paris cedex 05, France.

2 Interfaces, Confinement, Materiaux et Nanostructures, ICMN, UMR 7374, CNRS - Université d'Orléans, 45071 Orléans Cedex 02, France

*anne-laure.rollet@sorbonne-universite.fr ; jerome.fresnais@,sorbonne-unversite.fr

\section{Statement of article significance}

We investigate the NMR relaxation of iron oxide nanoparticles (MNPs). We sort by size MNPs to obtain numerous samples with diameter ranging from 4.5 to $12.5 \mathrm{~nm}$ with low polydispersity. We confirm that $r_{1}$ and $r_{2}$ NMR relaxivities increase with nanoparticle diameter. We also analyze the role of polydispersity for nanoparticles with the same mean size. Complementarily, we quantitatively investigate the role of coating on nanoparticles NMR relaxivity between bare and poly(sodium acrylate-co-maleate) coated nanoparticles (PAAMA). At last, we highlight that activation energy Ea decreases with nanoparticle diameter when determined from $T_{1}$, but increases for $T_{2}$ determination. 


\section{NMR relaxivity on coated and non-coated size-sorted maghemite nanoparticles}

Jérôme Fresnais ${ }^{1}$, QianQian $\mathrm{Ma}^{1}$, Linda Thai ${ }^{1}$, Patrice Porion ${ }^{2}$, Pierre Levitz $^{l}$ and Anne-Laure Rollet ${ }^{1} *$

${ }^{1}$ Sorbonne Université, CNRS, Laboratoire de Physico-chimie des Electrolytes et Nanosystèmes Interfaciaux, PHENIX - UMR 8234, F-75252 Paris cedex 05, France.

2 Interfaces, Confinement, Materiaux et Nanostructures, ICMN, UMR 7374, CNRS - Université d'Orléans, 45071 Orléans Cedex 02, France

* anne-laure.rollet@sorbonne-universite.fr ; jerome.fresnais@sorbonne-unversite.fr

\section{Supplementary information}

SI-1 : scheme of size sorting process

Size sorting process was achieved to obtain the more monodispersed MNPs batches

(Fig. SI-1a). From the original unsorted ferrofluid, 8 secondary batches are produced.

Batches 2SC, 4SC, 5SC, 6SC, 7SC, and 8SC are sorted again to reach different sizes with similar polydispersity. Sample 4SC9SC was sorted again to get $10.7 \mathrm{~nm}$ large MNPs, compared to 11.2 for 4SC9SC sample. 
Figure SI-1a : size sorting process of the first batch used in this study.

To reach very small nanoparticles, another batch was used and sorted thoroughly to have two sample denoted YP6SC and YP 7SC with diameter of $5.6 \mathrm{~nm}$ and $4.5 \mathrm{~nm}$, respectively (Fig. SI-1b).

\section{YP}

6SC

$75 C$

Figure SI-1b : size sorting process of the first batch used in this study.

SI-2 : properties of MNPs synthesized in this study

Table 1 : VSM, TEM, diameters with their correlated polydispersity (lognormal distribution) and DLS diameter of samples used in this study

\begin{tabular}{|c|c|c|c|c|c|}
\hline Uncoated MNPS & $\begin{array}{c}\text { VSM diameter } \\
(\mathrm{nm})\end{array}$ & $\sigma$ & $\begin{array}{c}\text { TEM diameter } \\
(\mathrm{nm})\end{array}$ & $\sigma$ & $\begin{array}{c}\text { DLS diameter } \\
(\mathrm{nm})\end{array}$ \\
\hline 4SCC & & & 12.72 & 0.29 & 28.8 \\
\hline 4SCSC & & & 11.88 & 0.28 & 26 \\
\hline 4SC9SC & 9.8 & 0.24 & 11.17 & 0.24 & 26.5 \\
\hline 5SC13SC & 7.8 & 0.16 & 9.4 & 0.17 & 6.2 \\
\hline 7SC3SC & 8.5 & 0.2 & 9.99 & 0.2 & 16.7 \\
\hline 8SCC & 8.6 & 0.21 & 9.23 & 0.21 & 15.5 \\
\hline
\end{tabular}




\begin{tabular}{|c|c|c|c|c|c|}
\hline 8SC4SC & 7.36 & 0.22 & 8.54 & 0.22 & 11.6 \\
\hline 8SC5SC & 7.4 & 0.19 & 7.73 & 0.19 & 12 \\
\hline 4SCS9C4SC & & & 10.68 & 0.17 & 15.3 \\
\hline 7SC12SC & & & 7.61 & 0.18 & 9.9 \\
\hline 6SC26SC & & & 7.21 & 0.18 & 9.4 \\
\hline 2SC31SC & & & 12.14 & 0.35 & \\
\hline YP1416SC & & & 5.6 & 0.25 & 7.8 \\
\hline YP1417SC & & & 4.5 & 0.16 & 9.2 \\
\hline & & & & & \\
\hline & & & TEM diameter & & DLS diameter \\
\hline Coated MNPS & VSM diameter & & $(\mathrm{nm})$ & & $(\mathrm{nm})$ \\
\hline 4SCC-PAAMA & & & 12.72 & 0.29 & 21.5 \\
\hline 4SCSC-PAAMA & & & 11.88 & 0.28 & 34.2 \\
\hline 4SC9SC-PAAMA & 9.8 & 0.24 & 11.17 & 0.24 & 34.7 \\
\hline 5SC13SC-PAAMA & 7.8 & 0.16 & 9.4 & 0.17 & 21.9 \\
\hline 7SC3SC-PAAMA & 8.5 & 0.2 & 9.99 & 0.2 & 22.6 \\
\hline 8SCC-PAAMA & 8.6 & 0.21 & 9.23 & 0.21 & 22 \\
\hline 8SC4SC-PAAMA & 7.36 & 0.22 & 8.54 & 0.22 & 20 \\
\hline 8SC5SC-PAAMA & 7.4 & 0.19 & 7.73 & 0.19 & 55.2 \\
\hline 4SC9SC4SC-PAAMA & & & 10.68 & 0.17 & 25.5 \\
\hline 2SC31SC-PAAMA & & & 12.14 & 0.35 & \\
\hline YP1416SC-PAAMA & & & 5.6 & 0.25 & 15.5 \\
\hline YP1417SC-PAAMA & & & 4.5 & 0.16 & 17.6 \\
\hline
\end{tabular}




\section{NMR relaxivity on coated and non-coated size-sorted maghemite nanoparticles}

Jérôme Fresnais ${ }^{1}$, QianQian Ma ${ }^{l}$, Linda Thai ${ }^{l}$, Patrice Porion ${ }^{2}$, Pierre Levitz $^{1}$ and Anne-Laure Rollet ${ }^{1} *$

${ }^{1}$ Sorbonne Université, CNRS, Laboratoire de Physico-chimie des Electrolytes et Nanosystèmes Interfaciaux, PHENIX - UMR 8234, F-75252 Paris cedex 05, France.

2 Interfaces, Confinement, Materiaux et Nanostructures, ICMN, UMR 7374, CNRS - Université d'Orléans, 45071 Orléans Cedex 02, France

* anne-laure.rollet@ sorbonne-universite.fr ; jerome.fresnais@ sorbonne-unversite.fr 


\section{NMR relaxivity on coated and non-coated size-sorted maghemite nanoparticles}

Relaxation dispersion profile, i.e. frequency dependence of the proton longitudinal relaxation times, were recorded for numerous samples of iron oxide nanoparticle dispersion with narrow size dispersity and diameters varying from 4 to $12.5 \mathrm{~nm}$. We demonstrated that $r_{1}$ and $r_{2}$ NMR relaxivities increase with nanoparticle diameter, as expected by the models. We also analyze the role of polydispersity for nanoparticles with the same mean size on the dispersion curves. Then, we compared intensively the role of coating on nanoparticles NMR relaxivity between bare and poly(sodium acrylate-co-maleate) coated nanoparticles. At last, we investigated the influence of nanoparticle size on the activation energy Ea. Interestingly, while Ea decreases with nanoparticle diameter when determined from $T_{1}$, it increases for $T_{2}$ determination. The influence is more important for small particles $(<9 \mathrm{~nm})$ than for big particles $(>9$ $\mathrm{nm})$. More, the PAAMA coating changes the energy Ea obtained from $T_{2}$ : Ea becomes independent of the nanoparticle diameters.

Keywords: maghemite nanoparticles; polymer coating; NMR relaxivity

\section{Introduction}

The magnetic nanoparticles (MNPs) are widely studied and used for their high efficiency as contrast agent in MRI combined with their therapeutic properties ${ }^{1-3}$. There are two major contributions to the relaxation of the magnetic moment of MNPs. First, their magnetic moment is proportional to the volume of the particles. The relaxation rates are thus largely ruled by the size of the particles, as well as by their anisotropy ${ }^{4}$. Second, the role of the coating on iron oxide nanoparticles is often neglected but can 
drastically modify the MNPs relaxation process ${ }^{5,6}$. Indeed, the coating can contribute to slow down the rotation of MNPs and modify the diffusion of solvent molecules compared to the medium. The relaxivity properties $r_{1}$ and $r_{2}$, i.e. the relaxation rate of the surrounding nuclei $R_{1}$ and $R_{2}$ divided by the concentration of iron, are the two parameters that are determined experimentally. They are usually obtained at a unique frequency, but more informations can be obtained by their determination at various frequencies ${ }^{7}$. Experimental results highlight that $r_{1}$ and $r_{2}$ varies with nanoparticle diameter ${ }^{8}$. The particle with the optimal diameter is thus an important parameter to be taken into account for bio-applications for magnetic resonance imaging (MRI) ${ }^{7}$. However, only few to no systematic experimental works were conducted to measure the relaxivity of iron oxide nanoparticles on a large range of particle size with controlled (and low) polydispersity. On the more, one important factor can impact the relaxivities of water in presence of MNPs. Indeed, it has also been evidenced that the aggregation state of the particle plays a crucial role in their relaxivity properties ${ }^{9-11}$. However, once incorporated in living animals, the MNPs may agglomerate or simply not be in a bulky state (adsorption on membrane cell...) ${ }^{12}$. Thus, the main recent developments are devoted to the design of efficient coatings that avoid or at least reduce magnetic dipolar interactions between MNPs ${ }^{3,13}$. Once again, the coating can modify significantly the relaxation process by slowing the rotation of the particles and perturbating the diffusion of solvent molecules in the vicinity of the MNPs.

Complementarily, the response depends on the nature of MNPs. The example of maghemite and cobalt ferrite MNPs is illustrative. The magnetic anisotropy energy constant $\mathrm{K}$ that reflects the way the magnetic moment of the MNPs fluctuates along the easy magnetic axes, is very different in these two cases ${ }^{14}$. For maghemite, its value is quite low (about $6 \mathrm{~kJ} / \mathrm{mol}$ ) and is ruled by surface effects ${ }^{15,16}$. For cobalt ferrite, its 
value is about 10 times higher than for maghemite and originate from the core of MNPs, surface effects being negligible. Hence, adsorption of molecules on maghemite MNPs might lead to important consequences on their relaxivity properties ${ }^{13}$. On the contrary, the cobalt ferrite MNPs relaxivity properties should be much less affected by coating.

In this article, we have been interested in the maghemite MNPs. The study of relaxivity properties is often hindered by polydispersity of MNPs batch that are produced and used in classical studies. Herein we focus on a drastic size sorting process to obtain large MNPs batches with controlled size and low polydispersity (less than 0.2 , according to a lognormal size distribution). We systematically compared the relaxivities of bare MNPs and poly(acrylate-co-maleate) coated nanoparticles, and explored the activation energy of uncoated and coated particles.

\section{Experimental and method}

\section{Maghemite nanoparticles synthesis and coating}

Iron oxide nanoparticles with bulk mass density $\rho=5.10^{3} \mathrm{~kg} \cdot \mathrm{m}^{-3}$ were synthesized according to the Massart's pathway by aqueous alkaline co-precipitation of iron (II) and iron (III) salts and oxidation of the magnetite $\left(\mathrm{Fe}_{3} \mathrm{O}_{4}\right)$ into maghemite $\left(\gamma-\mathrm{Fe}_{2} \mathrm{O}_{3}\right)$ to prevent further oxidation ${ }^{17}$. At $\mathrm{pH}=1.8$, the bare particles are positively charged, with nitrate counter-ions. They have a diameter size distribution centered on $8 \mathrm{~nm}$ according to a lognormal distribution. The resulting inter-particle interactions are repulsive and impart an excellent colloidal stability to the dispersion. However, the nanoparticle polydispersity is high (typically 0.45 following the lognormal distribution). As the magnetic properties are proportional to the volume of the nanoparticles, the polydispersity induce an average of the magnetic properties, thus the relaxivities. To 
highlight more precisely the influence of size nanoparticles on relaxivity, a size sorting has been achieved.

Size sorting is used to select sample with controlled size and lower size polydispersity. A well-established method was followed to obtain different sample with narrow size distribution ${ }^{18}$. Electrostatic repulsion between nanoparticles can be screened by the addition of salt. The repulsion will depend on the volume of the nanoparticles, and a phase separation is obtained where the concentrated phase contains the largest particles and the dilute one the smallest. Multiple steps of size sorting were achieved to obtain samples with the narrowest size distribution $(\sigma<0.2)$ and diameters going from $4.5 \mathrm{~nm}$ to $12 \mathrm{~nm}$ (see figure SI-1 for the size sorting pathway for the samples used in this study).

Experimentally, a given volume of nitric acid is added to the ferrofluid, that is placed on a magnet to separate the condensed phase $(\mathrm{C})$ from the supernatant $(\mathrm{S})$. The dense phase is washed with acetone and diethyl ether and redispersed in water. Another amount of nitric acid is added to the supernatant phase (S) and a second condensed phase (SC) is recovered and washed to obtain a second sample. The protocol is reproduced (samples $2 \mathrm{SC}, 3 \mathrm{SC} \ldots$ ) until the final supernatant is free from iron oxide nanoparticles. If the polydispersity of each sample is too large, each sample is sorted again by size following the same process. For instance, 5SC sorting processes give samples which are named 5SC1SC, 5SC2SC...

Ferrofluids were characterized with Vibrating Sample Magnetization (VSM), Transmission Electron Microscopy (TEM), atomic absorption spectrometry, and UVVisible spectrometry (see table 1 in SI-2 for complete informations about the samples used in this study). 
Bare nanoparticles were coated with poly(acrylic acid-co-maleic acid) polymer chains (PAAMA) using electrostatic interactions between carboxylate functions and the positive charges on the nanoparticles, similarly to a previous pathway used to coat MNPs with poly(sodium acrylate) ${ }^{19}$. PAAMA was purchase at Sigma Aldrich and was used without any purification. It consists of chains with average molecular weight of $3000 \mathrm{~g} / \mathrm{mol}$ with a molar ratio 1:1 between each monomers. A solution at $0.5 \%$ wt. of bare nanoparticles is added to a $0.5 \%$ wt. of PAAMA (1:2 volume ratio) at acidic $\mathrm{pH}$ value (1.8). A flocculation is induced by the interaction between the PAAMA and the nanoparticles. The transparent supernatant is removed and the $\mathrm{pH}$ of the condensed phase is increased up to 10 to redispersed the nanoparticles. This coating is particularly efficient to stabilize the nanoparticles in brine or complex media ${ }^{20}$.

\section{Maghemite nanoparticles characterization}

TEM is used to analyze physical size distribution of each nanoparticle sample. From different images, diameters of nanoparticles are measured. A statistic is achieved to obtain the size distribution of the nanoparticles. A lognormal distribution is used to fit the experimental data and obtain a mean diameter and a size polydispersity. This size is different from hydrodynamic diameter obtained by dynamic light scattering and the one obtained by VSM. This diameter resulting from the TEM image analysis is used to compare the relaxivity properties.

The concentration of ferrofluid are determined using both atomic absorption and UVvisible spectroscopies ${ }^{21}$. Both methods lead to comparable results.

Magnetic properties were obtained from a homemade VSM at room temperature, operating between -1 Tesla and +1 Tesla. From magnetization curves, magnetization at 
saturation and magnetic susceptometry were obtained. These values are used to modelize the relaxometry dispersion curves.

The measurements of the water ${ }^{1} \mathrm{H}$ relaxation times $\left(T_{1}\right.$ and $\left.T_{2}\right)$ were carried out on four different NMR apparatus. At $2 \mathrm{~T}\left(100 \mathrm{MHz}\right.$ for ${ }^{1} \mathrm{H}$ resonance frequency), the experiments were carried out using DSX100 Bruker spectrometer equipped with a diffusion probe (diff30 Bruker). The $T_{1}$ was measured using an inversion-recovery sequence and $\mathrm{T}_{2}$ using a Hahn echo, with $0.2 \mathrm{~s}$ recycle delay. At $1.2 \mathrm{~T}(60 \mathrm{MHz})$ and 0.4 $\mathrm{T}(20 \mathrm{MHz})$, the experiments were carried out using a 60 and a 20 Bruker Minispec, respectively. $T_{l}$ was measured using an inversion-recovery sequence with 16 recovery delays ranging from $40 \mu$ s to $10 T_{1}$ approximately. $T_{2}$ using a CPMG sequence with 50 to 500 echoes separated by $80 \mu \mathrm{s}$, a recycle delay of $0.2 \mathrm{~s}$.

The low frequency domain from $10 \mathrm{kHz}$ to $15 \mathrm{MHz}$ is explored on a Stelar Spin Master relaxometer. In this case, only $T_{1}$ has been measured using a PP sequence from $10 \mathrm{kHz}$ to $8 \mathrm{MHz}$ and a NP sequence from $8 \mathrm{MHz}$ to $15 \mathrm{MHz}{ }^{22}$.

The relaxivities $r_{1}=\left(T_{1}[\mathrm{Fe}]\right)^{-1}$ and $r_{2}=\left(T_{2}[\mathrm{Fe}]\right)^{-1}$ were determined using five MNPs dispersions with the iron concentration [Fe] ranging from 0.5 to $20 \mathrm{mM}$, approximately. In this range, the relaxation rates $R_{1}=1 / T_{1}$ and $R_{2}=1 / T_{2}$ vary linearly with [Fe], and the $r_{1}$ and $r_{2}$ values are obtained with a linear regression (figure $\mathrm{S} 1$ ). On the Stelar Spin Master, the measurements were performed for only one concentration because of the duration of the experiments. At $0.47 \mathrm{~T}$, the relaxivities $r_{1}$ and $r_{2}$ were measured as a function of five different temperatures $\left(\mathrm{T}=10,17,25,33\right.$ and $\left.40^{\circ} \mathrm{C}\right)$.

\section{Results and discussion}




\section{Effect of polydispersity}

The size polydispersity of the nanoparticles dispersion has an obvious effect on relaxivity as the latter is influenced by nanoparticles size and shape. To illustrate this influence, the $r_{1}$ and $r_{2}$ at $20 \mathrm{MHz}$ has been recorded for a large panel of maghemite samples and the $r_{l}$ profile has been recorded for two samples of the same medium size but with different polydispersity.

The polydispersity $\sigma$ of our samples was determined from the analysis of TEM images using ImageJ software and fitted using a log-normal function fitted over several thousand of MNPs.

$$
f(x ; \mu, \sigma)=\frac{1}{x \sigma \sqrt{2 \pi}} \exp \left(-\frac{(\ln x-\mu)^{2}}{2 \sigma^{2}}\right)
$$

The effect of polydispersity is first presented at one Larmor frequency $(20 \mathrm{MHz})$ in order to show its effect depending on the MNPs size. Figure 3 shows the value of $r_{1}$ and $r_{2}$ as a function of the MNPs size for several set of samples with different polydispersity: $\sigma<0.2, \sigma \approx 0.24, \sigma \approx 0.3, \sigma \approx 0.35$, and $\sigma>0.4$.

With no surprise, the greater size polydispersity, the greater $r_{1}$ and $r_{2}$ value dispersion. The effect is much more pronounced for $r_{1}$ than for $r_{2}$, for which the all points are relatively grouped. It can also be noticed that the increase of the polydispersity leads to lower relaxivity values for a given medium size. At first sight, it seems surprising because the increase of $\sigma$ in the log-normal function for a given medium size implies that the proportion of bigger MNPs is higher in the sample and an increase of the MNPs size leads to higher $r_{1}$ and $r_{2}$ relaxivities as shown in the same figure 3 .

In order to clarify this phenomenon, we have recorded the relaxation dispersion curve for two samples of the same medium size but with very different polydispersity: $d_{0}=10.8 \mathrm{~nm}$ with $\sigma=0.17$ and $\sigma=0.53$ i.e. non sorted sample (figure 4). For the 
maghemite sample with low polydispersity, the curve presents a well-defined profile with a plateau at low frequency, a depression just before the peak around $7 \mathrm{MHz}$ and the decrease at high frequency. For the maghemite sample with large polydispersity, the characteristic figure of maghemite dispersion profile is shaded. The depression before the peak has disappeared and the peak is somehow crushed, i.e. broader and less intense. Moreover, its position is shifted toward low frequency. Hence, it can also be noticed that at high frequency, i.e. around $20 \mathrm{MHz}$, it leads to a lower $r_{1}$ value as compared to the sample with low polydispersity.

\section{Effect of size}

We now present only the results for the highly sorted samples, i.e. for which the polydispersity is lower than 0.2 . Their NMR relaxivities $r_{1}$ et $r_{2}$ are plotted as a function of the TEM diameter in figure 5. An important increase is observed for both the longitudinal and the transversal components. The slope are about $6.4 \mathrm{~s}^{-1} \mathrm{mM}^{-1}$ per nm for $r_{1}$ and $17.8 \mathrm{~s}^{-1} \mathrm{mM}^{-1}$ per nm for $r_{2}$. This difference is due to the dependence of the difference in the Curie term for $r_{1}$ and for $r_{2}$.

Our values are well in line with the huge compilation study of Vuong et al. ${ }^{7}$ where the variation of $r_{2}$ with the size of MNPs has been studied over a very wide range of size (using also aggregates of MNPs). The authors show the linear dependence of $r_{2} / M_{v}$ with the particle diameter where $M_{v}$ is the saturation magnetization and this dependence pertains up to $0.4 \mu \mathrm{m}$.

The effect of size on $r_{1}$ is not usually studied because the targeted application for MNPs is $\mathrm{T}_{2}$ contrast agent. $r_{1}$ continuously increases with the diameter and tends to be linear in this range of size. This variation differs from the one observed for MNPs with high magnetic anisotropy constant like cobalt ferrite MNPs. In the latter case, $r_{1}$ increases with MNPs size but the slope decreases with the diameter. 
Tabletop relaxometer allows us to record rapidly the $r_{1}$ and $r_{2}$ and ergo to be able to study a lot of samples relaxivities. Considering the data discrepancies in the literature, it is crucial to get statistics to investigate parameters like size, coating etc. This is an obvious advantage of this kind of relaxometers. However, the effect of the various parameters is better revealed by NMR relaxation profile.

On figure 6 are presented the $r_{1}$ profiles for non-coated MNPs with $(\sigma \leq 0.2)$ and with diameter ranging from 4 to $11 \mathrm{~nm}$. By increasing the size of spherical superparamagnetic nanoparticles several features occurs in the $r_{1}$ profile: (a) the maximum of the bump is shifted toward lower frequency, (b) the height of this maximum is increased, (c) the value of the plateau at low frequency is increased and (d) the hollow just before the bump is decreased. As already explained by Roch et al. ${ }^{23}$ in their model, these variations are the result of a balance between the different dynamical characteristic times of the system (Neel time, diffusion time, rotational time).

\section{Effect of coating}

The PAAMA coating is achieved through electrostatic anchoring of the carboxylate functions at the nanoparticle surfaces. The molecular weight of the PAAMA chains $(3,000 \mathrm{~g} / \mathrm{mol})$ corresponds to qualitatively 16 equivalent monomers of acrylate-maleate. This corresponds to a total length of the polymer chain of qualitatively 10-12 nm when completely elongated. Part of the monomers is adsorbed on the surface of the nanoparticles, and the chains are not completely straight in solution. This reduced drastically the thickness of the PAAMA corona around nanoparticles. Dynamic light scattering experiment comparing bare and PAAMA coated MNPs demonstrated an 
increase of diameter of 4-5 $\mathrm{nm}$ for almost all MNPs batches, that is in good agreement with what was observed for poly(sodium acrylate) coated nanoparticles ${ }^{24}$.

Bare and PAAMA coated MNPs were characterized by VSM. Curves between uncoated and coated MNPs are superimposed, and give same diameters and polydispersities before and after coating. This shows that the coating has no influence of the magnetic behavior of MNPs against applied magnetic field.

The values of $r_{1}$ and $r_{2}$ for PAAMA coated MNPs at $20 \mathrm{MHz}$ are compared with those of non-coated MNPs (figure 5). The diameter considered here is the maghemite diameter without the polymer corona. The values are clearly superimposed for the $r_{2}$ while for the $r_{l}$ for PAAMA coated MNPs are slightly below the $r_{l}$ for non-coated MNPs. It can be noted that the effect of coating on the $r_{1}$ and $r_{2}$ at high frequency is subject to high discrepancies of partially and uncontrolled MNPs aggregation phenomena ${ }^{25}$. Hence, the $r_{1}$ profiles is more able to reveal the coating effect.

The $r_{l}$ profiles have been recorded for PAAMA coated MNPs and plotted in figure 6. The evolution of the curves with the MNPs diameter is very similar to the curves for non-coated MNPs. In order to better reveal the effect of the PAAMA coating, the profile for non-coated and PAAMA coated MNPs have been superimposed for four sizes, 5.6, 7.7, 10.0 and $12.7 \mathrm{~nm}$ in figure 7 . Several observations can be made when the MNPs are coated: (a) the value of the plateau at low frequency is smaller; (b) the hollow before the bump is less pronounced; (c) the position of the hollow is shifted toward low frequency; (d) the position of the bump is not shifted; (e) the bump maximum is affected and (f) the profiles tend to merge at high frequency.

To illustrate these effects, we have also plotted in figure 8 the ratio $A$ between the maximum of $r_{l}\left(r_{l}{ }^{\max }\right)$ and the $r_{l}$ at $10 \mathrm{kHz}$ (the plateau value $r_{l}^{\text {plateau }}$ ) and the ratio $B$ 
between the minimum of $r_{l}$ before the bump $\left(r_{l}{ }^{\text {deep }}\right)$ and the $r_{l}{ }^{\text {plateau }}$. From figure 8 it is clear that the height associated to the Curie term in the models ${ }^{26}$ is increased with the coating. It can be also notice that the difference between the ratio $A$ for non-coated and coated MNPs is roughly constant with the maghemite diameter. The striking point is that the position of the bump is not affected in the same time. The models predict a combined modification of the bump height and its position ${ }^{14,23}$. As illustrated by Kruk et al. ${ }^{27}$ in maghemite MNPs dispersed in decaline and in toluene, a slowdown of the solvent dynamics lead to both the decrease of the bump and the shift of its position toward low frequencies. In the latter case, two solvents of different viscosities were used. In the case of coated MNPs, only the dynamics of water inside the polymer corona and in the very close vicinity is modified (along with the dynamics of the whole MNPs). To modelize the relaxivity of polymer coated MNPs it is therefore necessary to reconsider the diffusion propagator of water with a two steps environments. On figure 8 , we have also plotted the ratio $B$ between the minimum of $r_{1}$ before the bump $\left(r_{1}^{\text {deep }}\right)$ and the $r_{l}{ }^{\text {plateau }}$. Here again a clear feature can be observed.

The MNPs in the models are impenetrable sphere and the diffusion of water is homogenous whatever the distance to the MNPs. In the case of polymer coated MNPs there is a corona around the MNPs where the diffusion of water is hindered. The effect of the modification of water diffusion in the vicinity of MNPs has been underlined by Ye et al. ${ }^{28}$ who compared the relaxivities of magnetite coated by silica or capped by cetyltrimethyl ammonium bromide. 


\section{Activation energy}

The relaxivity $r_{1}$ and $r_{2}$ at $20 \mathrm{MHz}$ have been measured at different temperatures ranging from 10 to $40^{\circ} \mathrm{C}$. In this range, the relaxivities exhibit an Arrhenius behavior and it was possible to determine an activation energy Ea. It must be underlined that at $20 \mathrm{MHz}$ the relaxivity is ruled by the Curie term. The latter is related to the characteristic time $\tau_{D}=d^{2} / D$ of the water diffusion around the MNPs ( $d$ is the diameter of the MNPs and $D$ is the self-diffusion coefficient of water). The energy Ea values obtained are in the same range of Ea for bulk water $(19 \mathrm{~kJ} / \mathrm{mol})^{29}$. The results are presented in figure 9 as function of the maghemite size. For the non-coated MNPs, the first striking point is the difference of behaviour in the Ea variations for $r_{1}$ and for $r_{2}$. For $r_{1}$ Ea is constant for small MNPs approximately up to $10 \mathrm{~nm}$, and then decreases. The behavior is opposite for $r_{2} \mathrm{Ea}$ as the latter increases up to $10 \mathrm{~nm}$ and then is roughly constant. It must be stressed also that these variations are very different from those observed for MNPs with high magnetic anisotropy. In the case of cobalt ferrite MNPs Ea of both the $r_{1}$ and the $r_{2}$ decreases. These differences are very interesting because MNPs with high (cobalt ferrite) and low (maghemite) magnetic anisotropy energy have very different $r_{1}$ profiles, but above $20 \mathrm{MHz}$, their $r_{1}$ and $r_{2}$ values are very similar. Therefore, the only measurement of Ea for $r_{1}$ and the $r_{2}$ relaxivities using a tabletop relaxometer is not sufficient to investigate the magnetic anisotropy effects. The measurement of Ea of $r_{1}$ and $r_{2}$ thus opens an opportunity for such studies and furthermore for studies on the tuning of the anisotropy properties.

The coating influences slightly the $r_{1}$ and the $r_{2}$ activation energy. For small size MNPs, Ea is increased by the coating while no effect is observed for larger maghemite $(>8 \mathrm{~nm})$. Greater effect was expected as the Ea, at this frequency, is dominated by the Curie term, 
i.e. correlated with the $\tau_{\mathrm{D}}$ time. Hence the polymer corona seems to affect the relaxivities properties only for small maghemite MNPs.

To analyze more precisely the impact of the coating on the activation energy, we can evaluate the role of surface to volume ratio, that is proportional to the inverse of the particle diameter. Indeed, we should be able to highlight the role of the coating on the relaxation process. Thus, we plotted the ratio between activation energies Ea (measure either on $\mathrm{T}_{1}$ or $\mathrm{T}_{2}$ ) of coated and uncoated particles versus the inverse of the particles diameter (figure 10).

There are clearly some dispersions in measurements, but it can be seen that the activation energy ratio measured on $T_{l}$ values are mainly larger than unity, and increases with the inverse of the diameter. The activation energy ratio values obtained from $T_{2}$ measurement shows an increase with increasing value of $1 / \mathrm{D}$ as well, as observed with Ea values measured from $T_{1}$. These results show that the coating on the nanoparticles play a significant role in the relaxation process of water molecules in the polymer corona compared to bare MNPs.

\section{Conclusion}

The influences of the size, the sample polydispersity and the polymer coating of maghemite nanoparticles on NMR relaxivities have been investigated. In agreement with previous studies, $r_{1}$ and $r_{2}$ increase with the MNPs diameter. The NMR relaxation profile better reveal the influence of the size with a shift of the $r_{1}$ maximum toward low frequency. It is shown the importance of controlling the size of the sample as in addition to higher discrepancy in $r_{1}$ and $r_{2}$ values, the NMR relaxation profile is significantly changed with a general broadening and an important shift of the $r_{l}$ maximum toward 
low frequency. Polymer coating influences the relaxivities of maghemite MNPs as shown by the NMR relaxation profiles. The most striking and surprising feature is that the height of the bump is modified while its position is unchanged. The activation energy of $T_{1}$ and $T_{2}$ have been measured at $20 \mathrm{MHz}$ for non-coated and coated maghemite MNPs. This activation energy Ea exhibits an interesting dependence on the magnetic properties as its variation with MNPs size is clearly different for maghemite and cobalt ferrite MNPs, which magnetic anisotropy energy is ten time higher in the latter case than in the former case. The Ea of maghemite MNPs is influenced by the polymer coating only for small size MNPs $(<8 \mathrm{~nm})$.

\section{Aknowledgement}

The authours acknowledge COST Action CA15209 EURELAX "European Network on NMR Relaxometry", supported by COST (European Cooperation in Science and Technology).

\section{References}

${ }^{1}$ G. Béalle, R. Di Corato, J. Kolosnjaj-Tabi, V. Dupuis, O. Clément, F. Gazeau, C.

Wilhelm, and C. Ménager, Langmuir 28, 11834 (2012).

${ }^{2}$ S.A. Corr, S.J. Byrne, R. Tekoriute, C.J. Meledandri, D.F. Brougham, M. Lynch, C.

Kerskens, L. O’Dwyer, and Y.K. Gun'ko, J. Am. Chem. Soc. 130, 4214 (2008).

${ }^{3}$ A.K. Gupta and M. Gupta, Biomaterials 26, 3995 (2005).

${ }^{4}$ A.-L. Rollet, S. Neveu, P. Porion, V. Dupuis, N. Cherrak, and P. Levitz, Phys. Chem. Chem. Phys. 18, 32981 (2016).

${ }^{5}$ S. Kachbi-Khelfallah, M. Monteil, M. Cortes-Clerget, E. Migianu-Griffoni, J.-L. Pirat, O. Gager, J. Deschamp, and M. Lecouvey, Beilstein Journal of Organic Chemistry 12, 
1366 (2016).

${ }^{6}$ E. Umut, in Modern Surface Engineering Treatments, edited by M. Aliofkhazraei (InTech, 2013).

${ }^{7}$ Q.L. Vuong, J.-F. Berret, J. Fresnais, Y. Gossuin, and O. Sandre, Advanced Healthcare Materials 1, 502 (2012).

${ }^{8}$ M.-S. Martina, J.-P. Fortin, C. Ménager, O. Clément, G. Barratt, C. GrabielleMadelmont, F. Gazeau, V. Cabuil, and S. Lesieur, J. Am. Chem. Soc. 127, 10676 (2005).

${ }^{9}$ I.Y. Tóth, D. Nesztor, L. Novák, E. Illés, M. Szekeres, T. Szabó, and E. Tombácz, Journal of Magnetism and Magnetic Materials 427, 280 (2017).

${ }^{10}$ E. Peng, F. Wang, and J.M. Xue, J. Mater. Chem. B 3, 2241 (2015).

${ }^{11}$ B.A. Larsen, M.A. Haag, N.J. Serkova, K.R. Shroyer, and C.R. Stoldt, Nanotechnology 19, 7 (2008).

${ }^{12}$ R. Di Corato, A. Espinosa, L. Lartigue, M. Tharaud, S. Chat, T. Pellegrino, C. Ménager, F. Gazeau, and C. Wilhelm, Biomaterials 35, 6400 (2014).

${ }^{13}$ L.E.W. LaConte, N. Nitin, O. Zurkiya, D. Caruntu, C.J. O’Connor, X. Hu, and G. Bao, Journal of Magnetic Resonance Imaging 26, 1634 (2007).

${ }^{14}$ A. Rollet, S. Neveu, P. Porion, V. Dupuis, N. Cherrak, and P. Levitz, Physical Chemistry Chemical Physics: PCCP (2016).

${ }^{15}$ C. Guibert, J. Fresnais, V. Peyre, and V. Dupuis, Journal of Magnetism and Magnetic Materials 421, 384 (2017).

${ }^{16}$ F. Gazeau, J.C. Bacri, F. Gendron, R. Perzynski, Y.L. Raikher, V.I. Stepanov, and E. Dubois, Journal of Magnetism and Magnetic Materials 13 (1998).

${ }^{17}$ R. Massart and V. Cabuil, Journal De Chimie Physique Et De Physico-Chimie Biologique 84, 967 (1987). 
${ }^{18}$ S. Lefebure, E. Dubois, V. Cabuil, S. Neveu, and R. Massart, Journal of Materials Research 13, 2975 (1998).

${ }^{19}$ A. Sehgal, Y. Lalatonne, J.F. Berret, and M. Morvan, Langmuir 21, 9359 (2005).

${ }^{20}$ C. Guibert, V. Dupuis, V. Peyre, and J. Fresnais, J. Phys. Chem. C 119, 28148 (2015).

${ }^{21}$ C. Guibert, V. Dupuis, J. Fresnais, and V. Peyre, Journal of Colloid and Interface Science 454, 105 (2015).

${ }^{22}$ E. Anoardo, G. Galli, and G. Ferrante, Applied Magnetic Resonance 20, 365 (2001).

${ }^{23}$ A. Roch, R.N. Muller, and P. Gillis, The Journal of Chemical Physics 110, 5403 (1999).

${ }^{24}$ J. Fresnais, M. Yan, J. Courtois, T. Bostelmann, A. Bée, and J.-F. Berret, Journal of Colloid and Interface Science 395, 24 (2013).

${ }^{25}$ Matthew R J Carroll and Phillip P Huffstetler and William C Miles and Jonathon D Goff and Richey M Davis and Judy S Riffle and Michael J House and Robert C Woodward and Timothy G St Pierre, Nanotechnology 22, 325702 (2011).

${ }^{26}$ M. Lévy, F. Gazeau, C. Wilhelm, S. Neveu, M. Devaud, and P. Levitz, J. Phys. Chem. C 117, 15369 (2013).

${ }^{27}$ D. Kruk, A. Korpała, S.M. Taheri, A. Kozłowski, S. Förster, and E.A. Rössler, The Journal of Chemical Physics 140, 174504 (2014).

${ }^{28}$ Ye Fei, Laurent Sophie, Fornara Andrea, Astolfi Laura, Qin Jian, Roch Alain, Martini Alessandro, Toprak Muhammet S., Muller Robert N., and Muhammed Mamoun, Contrast Media \& Molecular Imaging 7, 460 (2012).

${ }^{29}$ J.H. Simpson and H.Y. Carr, Phys. Rev. 111, 1201 (1958). 

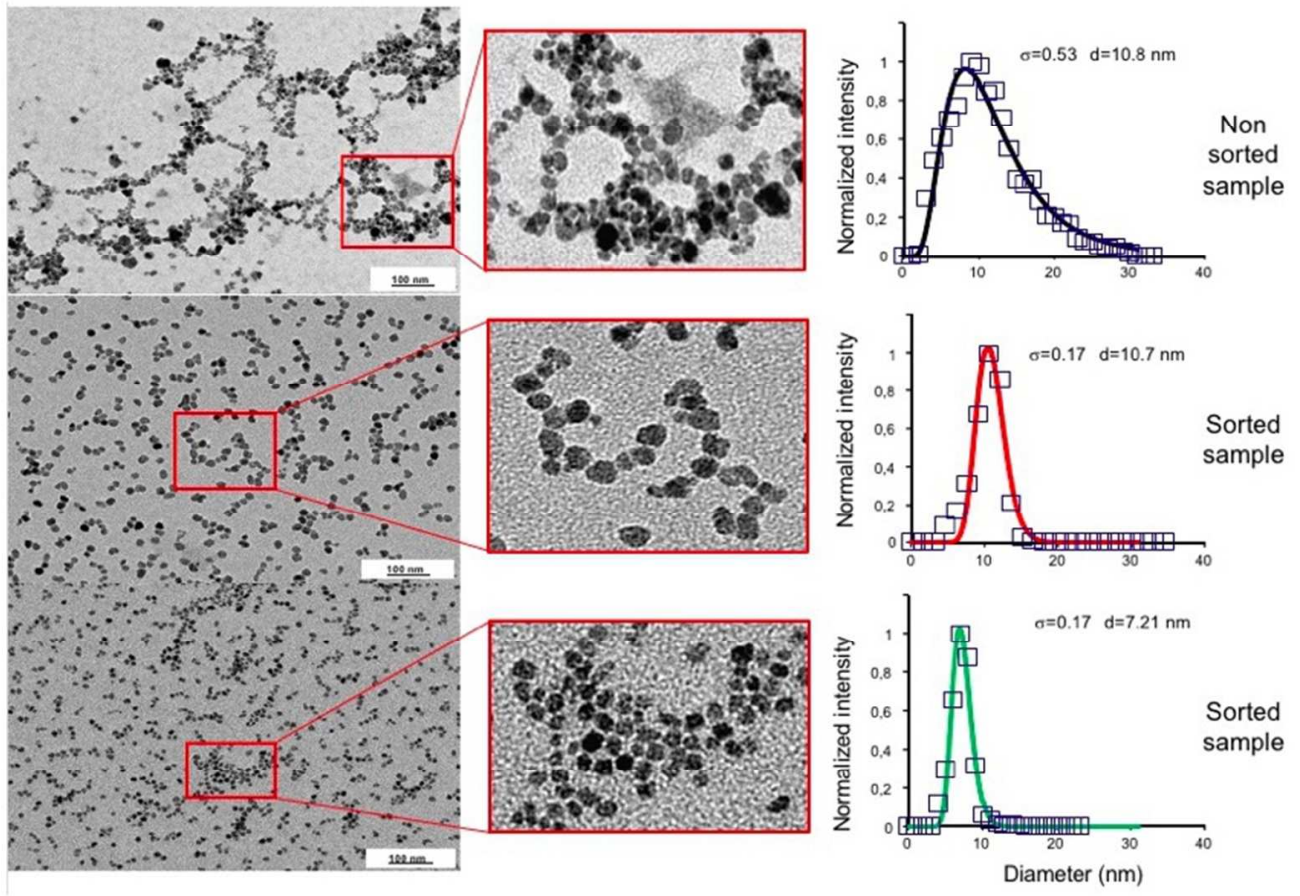

Figure 1: TEM image of the bare maghemite nanoparticles before sorting and after sorting process.

$300 \times 207 \mathrm{~mm}(72 \times 72 \mathrm{DPI})$ 
Figure 2: scheme of the bare maghemite nanoparticles (left) and PAAMA coated MNPs (right). $309 \times 120 \mathrm{~mm}(72 \times 72 \mathrm{DPI})$ 

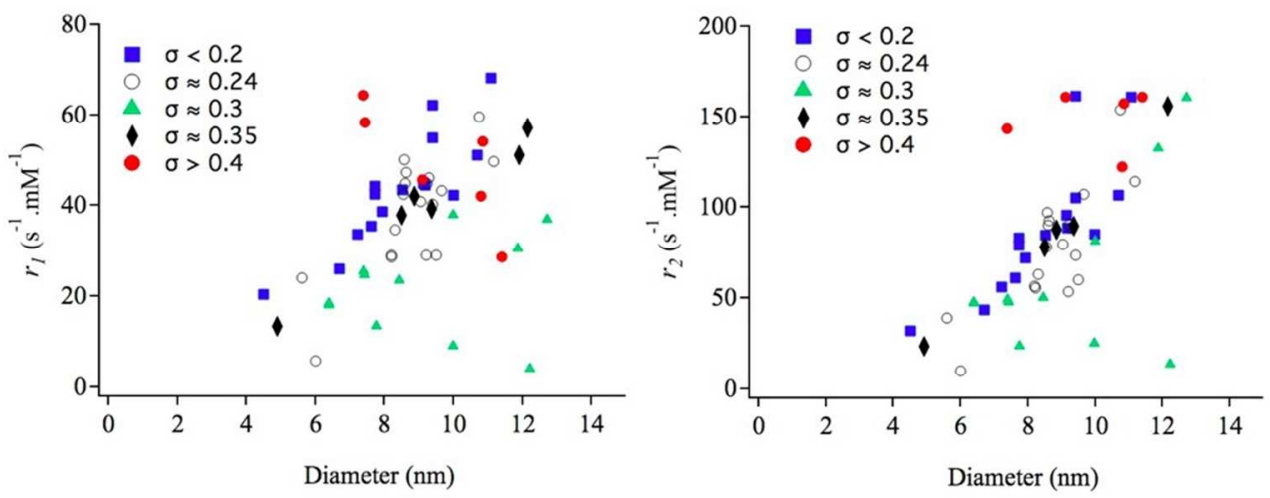

Figure 3: effect of the polydispersity $\sigma$ on $\mathrm{r} 1$ and $\mathrm{r} 2: \sigma<0.2$ (blue squares), $\sigma \approx 0.24$ (empty circles), $\sigma \approx$ 0.3 (green triangles), $\sigma \approx 0.35$ (black diamonds), and $\sigma>0.4$ (red disks).

\section{$366 \times 141 \mathrm{~mm}(72 \times 72$ DPI $)$}




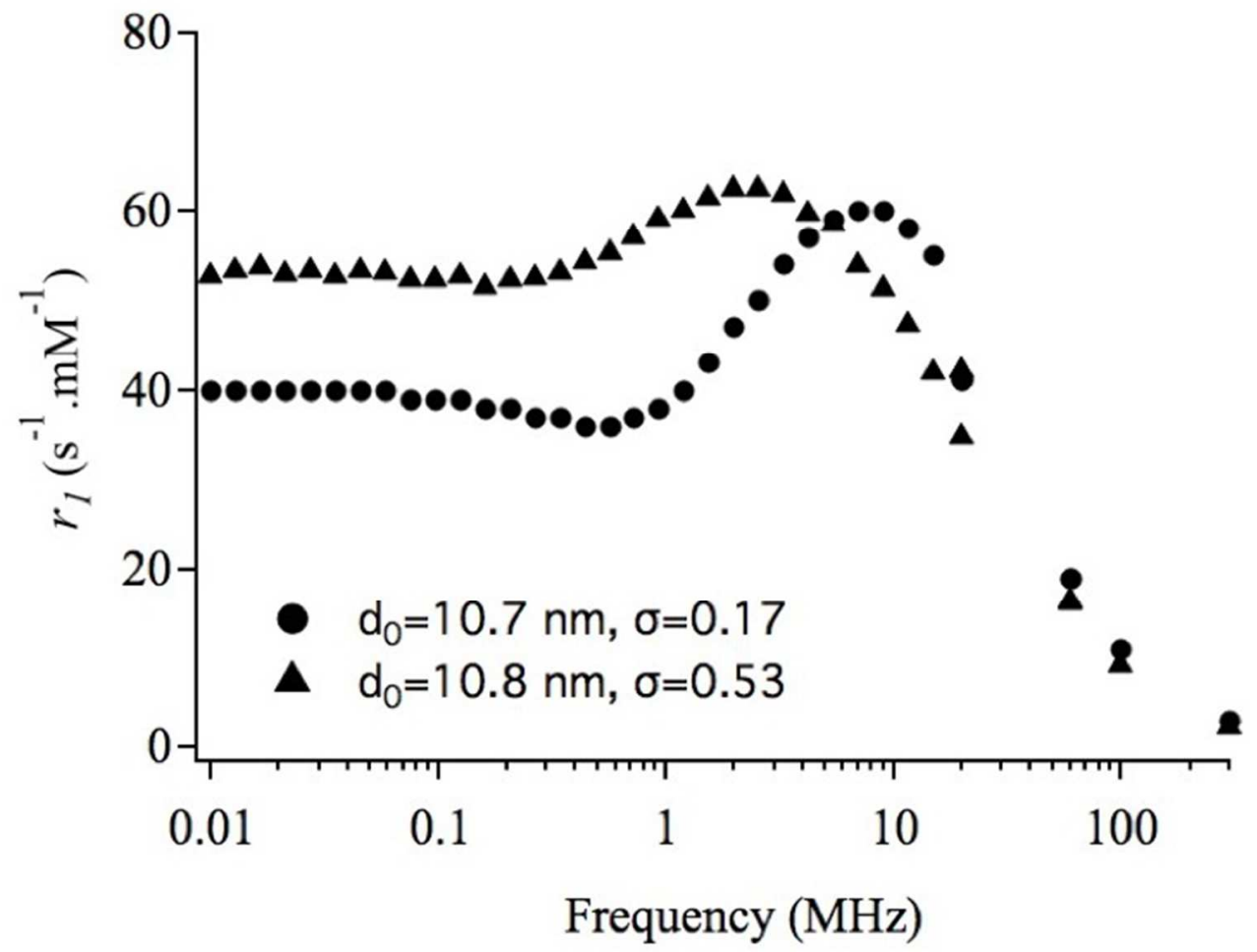

Figure 4: r1 NMRD profile for two samples of the same medium size but with very different polydispersity: $d$ $=10.7 \mathrm{~nm}$ with $\sigma=0.17$ (circle) and $\mathrm{d}=10.8 \mathrm{~nm} \sigma=0.53$ (triangle).

$262 \times 201 \mathrm{~mm}(72 \times 72 \mathrm{DPI})$ 

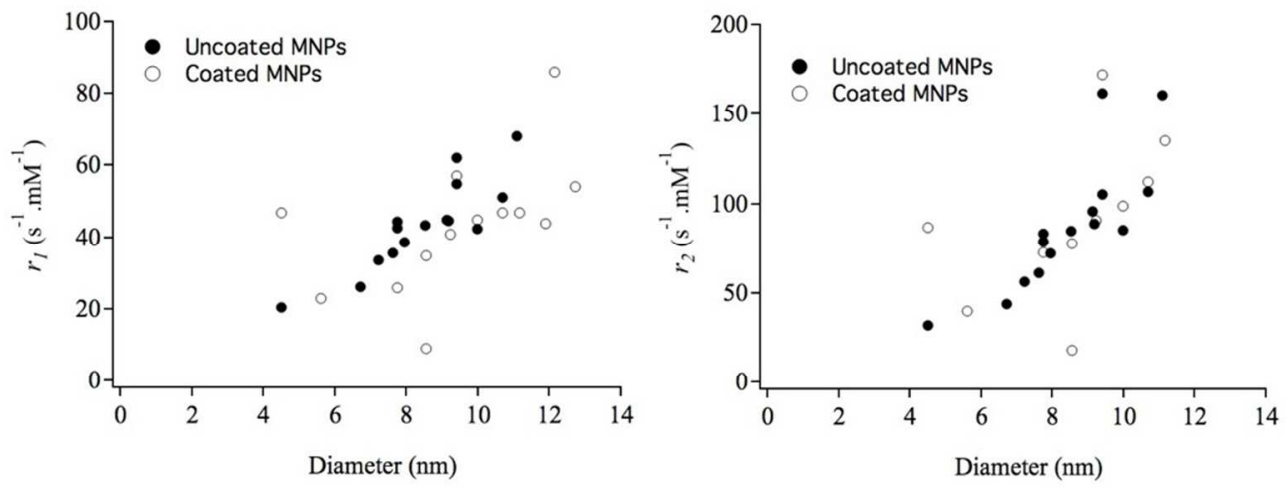

Figure 5: $\mathrm{r} 1$ and $\mathrm{r} 2$ relaxivities of uncoated (black symbol) and coated (empty symbol) maghemite as a function of the diameter (for $\sigma \leq 0.2$ ). $367 \times 149 \mathrm{~mm}(72 \times 72 \mathrm{DPI})$ 

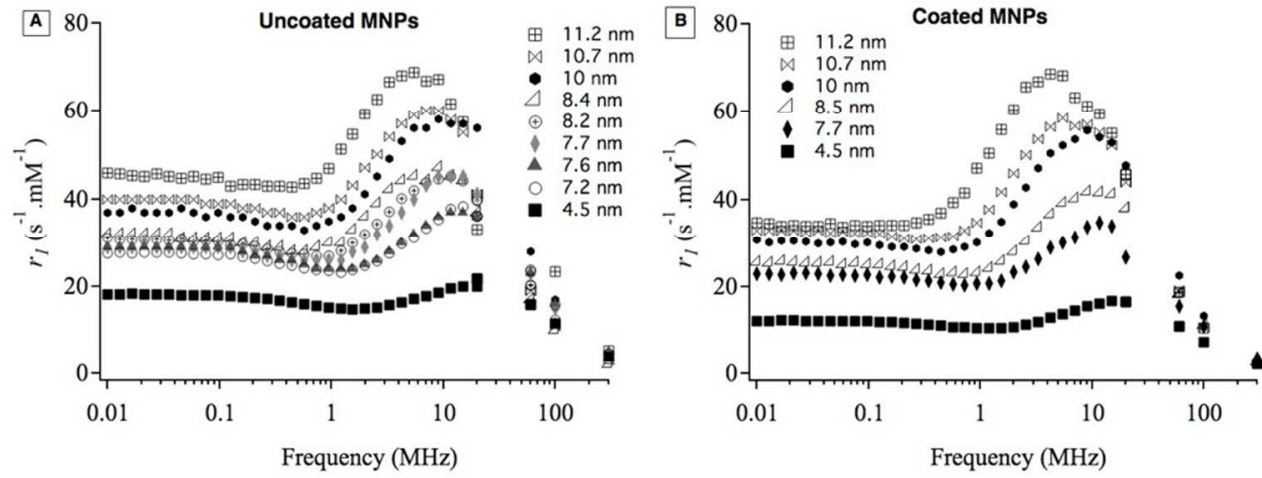

Figure 6: $r 1$ profile of maghemite uncoated MNPs (A) and PAAMA-coated MNPs (B) for diameters of the maghemite core (polymer corona not included) ranging from 4.5 to $11.2 \mathrm{~nm}$.

$368 \times 141 \mathrm{~mm}(72 \times 72$ DPI $)$ 


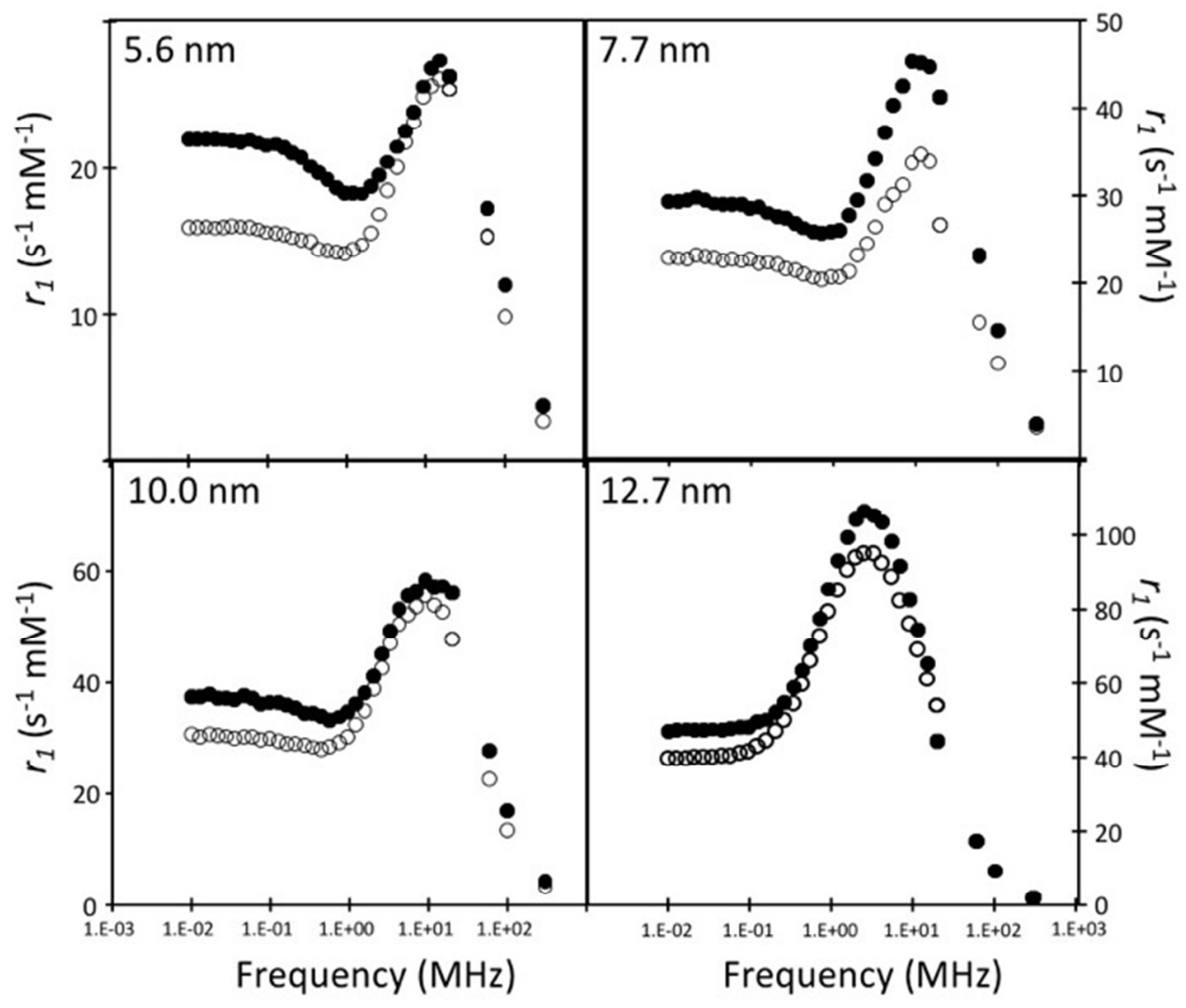

Figure 7: r1 profile of maghemite MNPs non coated (black symbol) and PAAMA coated (empty symbol) for several diameters of the maghemite core (polymer corona not included): 5.6, 7.7, 10 and $12.7 \mathrm{~nm}$.

$$
254 \times 213 \mathrm{~mm}(72 \times 72 \text { DPI })
$$



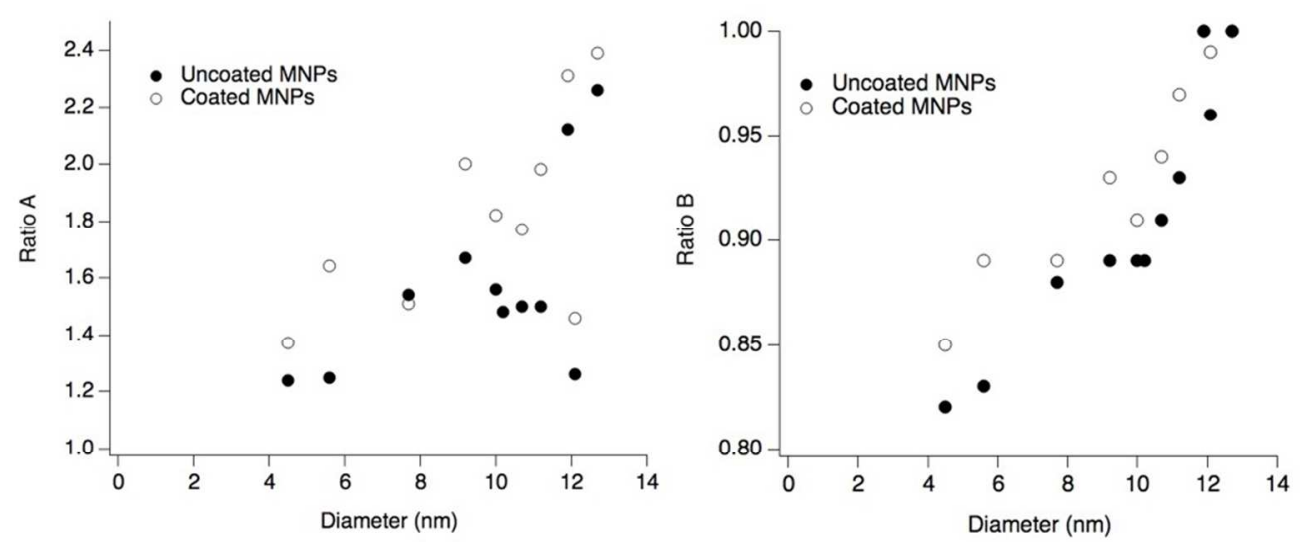

Figure 8: ratio A between the maximum of $\mathrm{r} 1$ ( $\mathrm{r} 1 \mathrm{max}$ ) and the $\mathrm{r} 1$ at $10 \mathrm{kHz}$ (the plateau value r1plateau) and the ratio $\mathrm{B}$ between the minimum of $\mathrm{r} 1$ before the bump ( $r$ 1deep) and the r1plateau as a function of the maghemite diameter for uncoated (black disks) and coated (empty circles) MNPs.

\section{$361 \times 155 \mathrm{~mm}(72 \times 72 \mathrm{DPI})$}


Figure 9: Activation energy Ea of T1 (left) and T2 (right) Ea for non-coated (black symbol) and coated (empty symbol) maghemite as a function of the diameter.

$368 \times 147 \mathrm{~mm}(72 \times 72 \mathrm{DPI})$ 

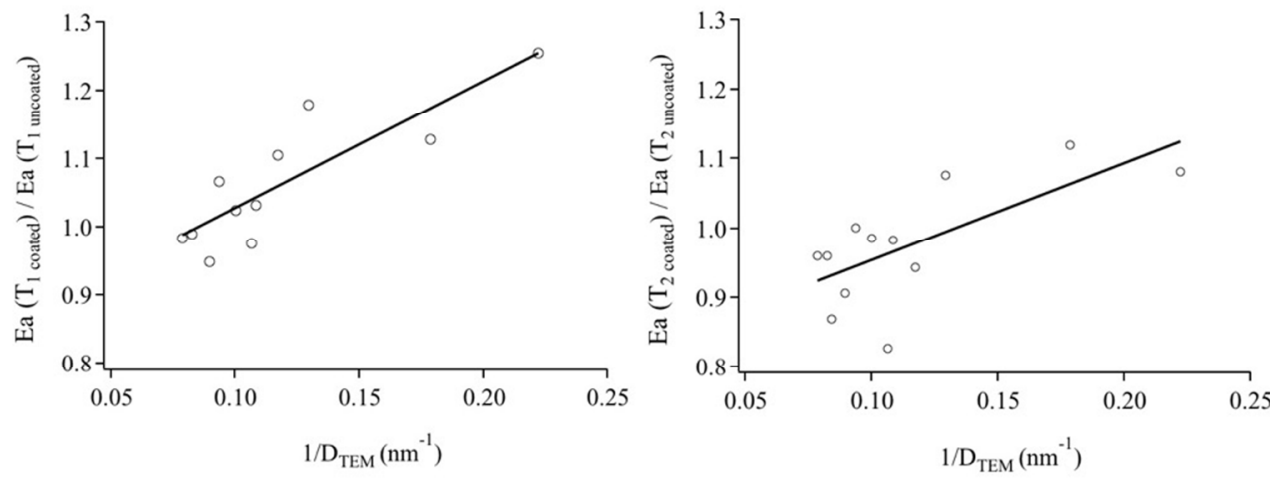

Figure 10: ratio of activation energy Ea of T1 (left) and T2 (right) Ea between non-coated and coated maghemite as a function of the inverse of the diameter. Lines are linear fits.

\section{$366 \times 143 \mathrm{~mm}(72 \times 72 \mathrm{DPI})$}




\section{NMR relaxivity on coated and non-coated size-sorted maghemite nanoparticles}

Jérôme Fresnais ${ }^{1 *}$, QianQian Ma ${ }^{l}$, Linda Thai ${ }^{1}$, Patrice Porion ${ }^{2}$, Pierre Levitz ${ }^{1}$ and Anne-Laure Rollet ${ }^{1 *}$

1 Sorbonne Université, CNRS, Laboratoire de Physico-chimie des Electrolytes et Nanosystèmes Interfaciaux, PHENIX - UMR 8234, F-75252 Paris cedex 05, France.

2 Interfaces, Confinement, Materiaux et Nanostructures, ICMN, UMR 7374, CNRS - Université d'Orléans, 45071 Orléans Cedex 02, France

* anne-laure.rollet@sorbonne-universite.fr ; jerome.fresnais@,sorbonne-unversite.fr

\section{Figures}
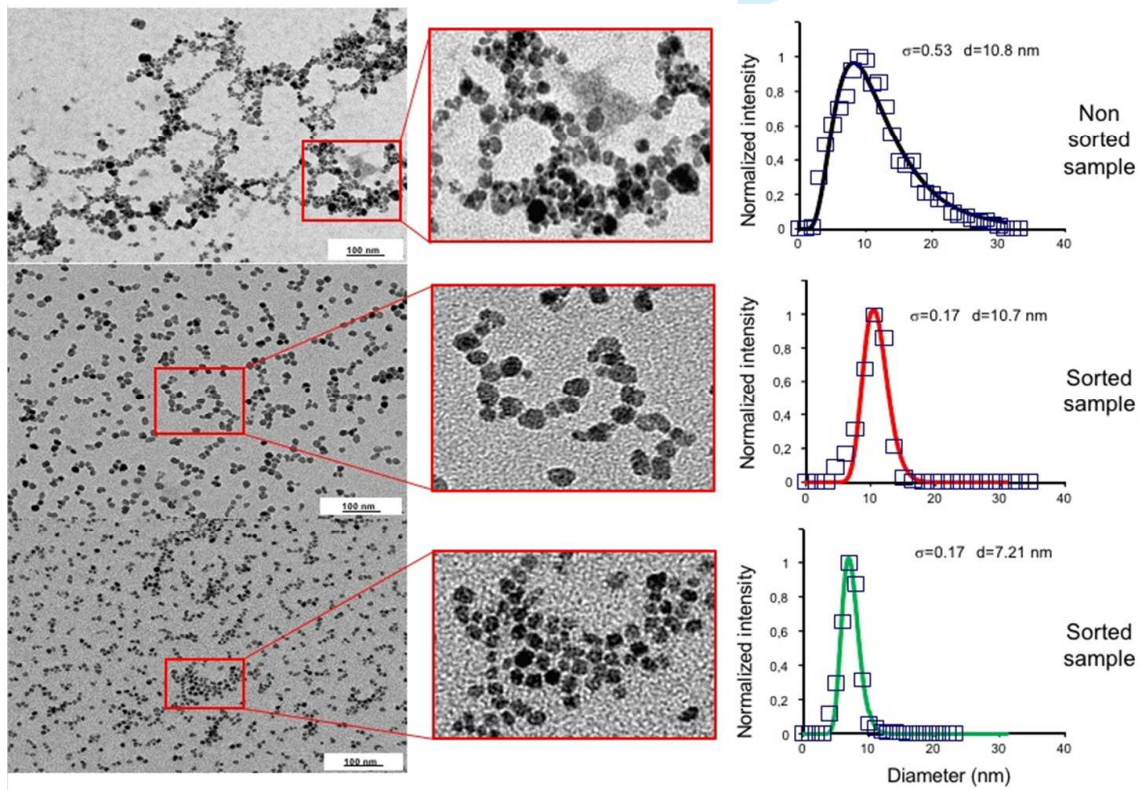
Figure 1: TEM image of the bare maghemite nanoparticles before sorting and after sorting process.

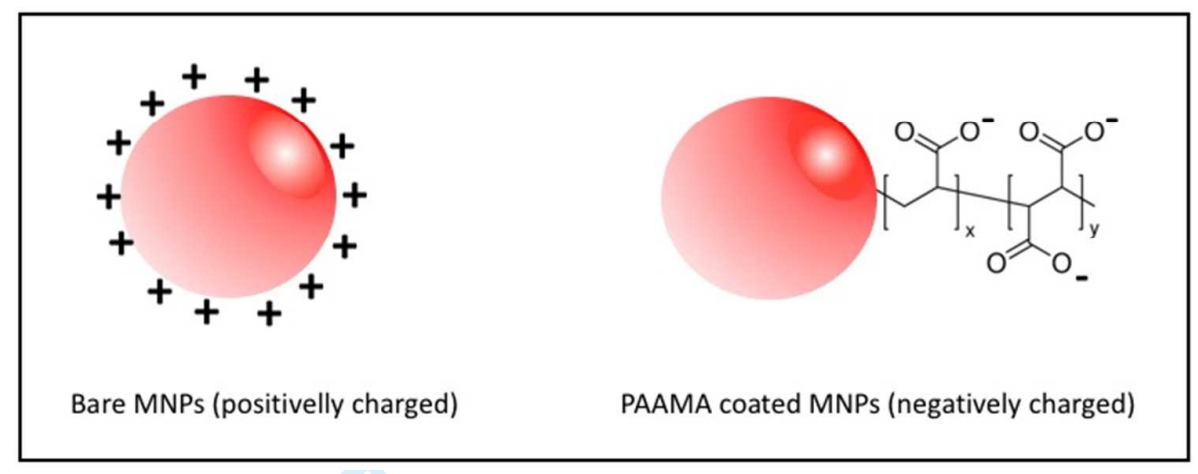

Figure 2: scheme of the bare maghemite nanoparticles (left) and PAAMA coated MNPs (right).
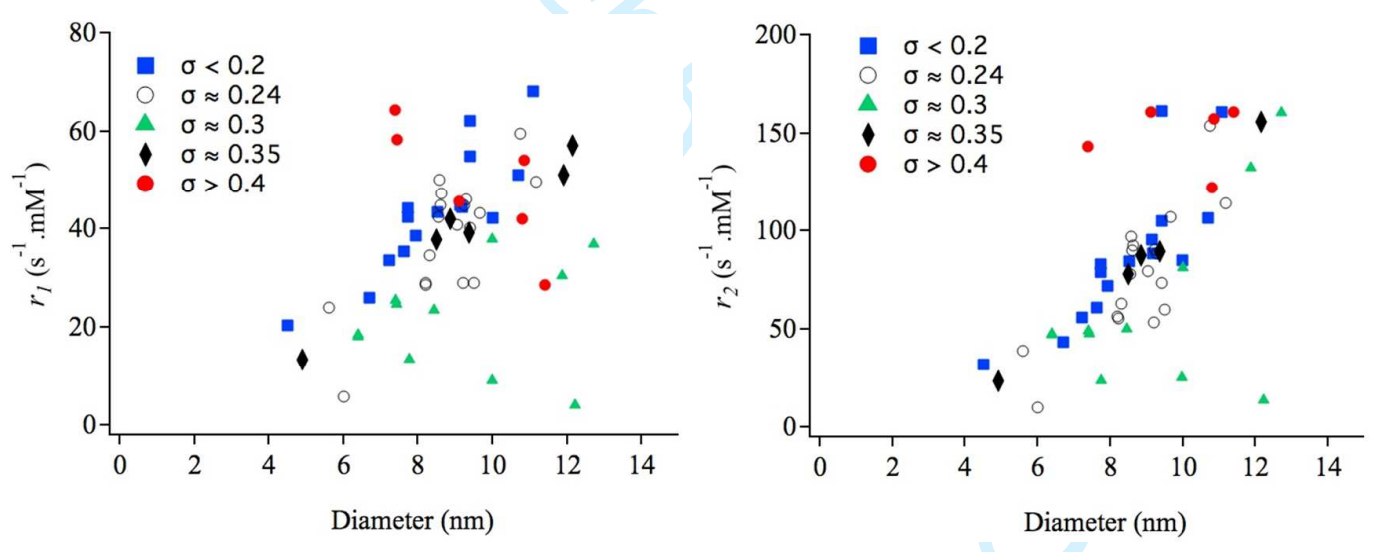

Figure 3: effect of the polydispersity $\sigma$ on $r_{1}$ and $r_{2}: \sigma<0.2$ (blue squares), $\sigma \approx 0.24$ (empty circles), $\sigma \approx 0.3$ (green triangles), $\sigma \approx 0.35$ (black diamonds), and $\sigma>0.4$ (red disks). 


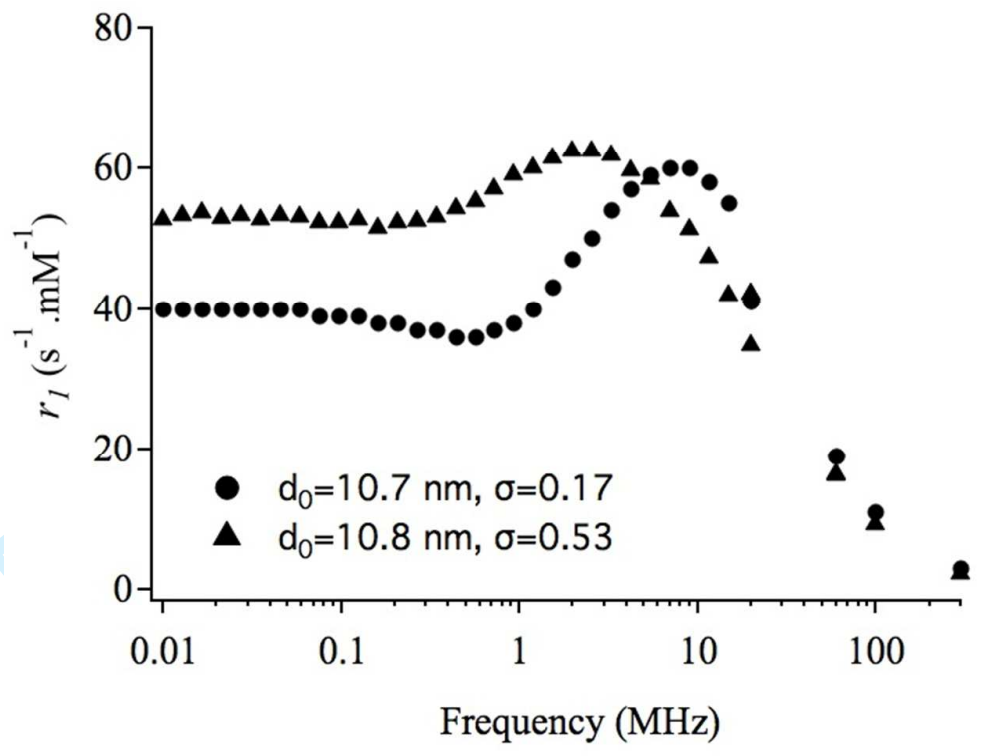

Figure 4: $r_{l}$ NMRD profile for two samples of the same medium size but with very different polydispersity: $d=10.7 \mathrm{~nm}$ with $\sigma=0.17$ (circle) and $d=10.8 \mathrm{~nm} \sigma=0.53$ (triangle).
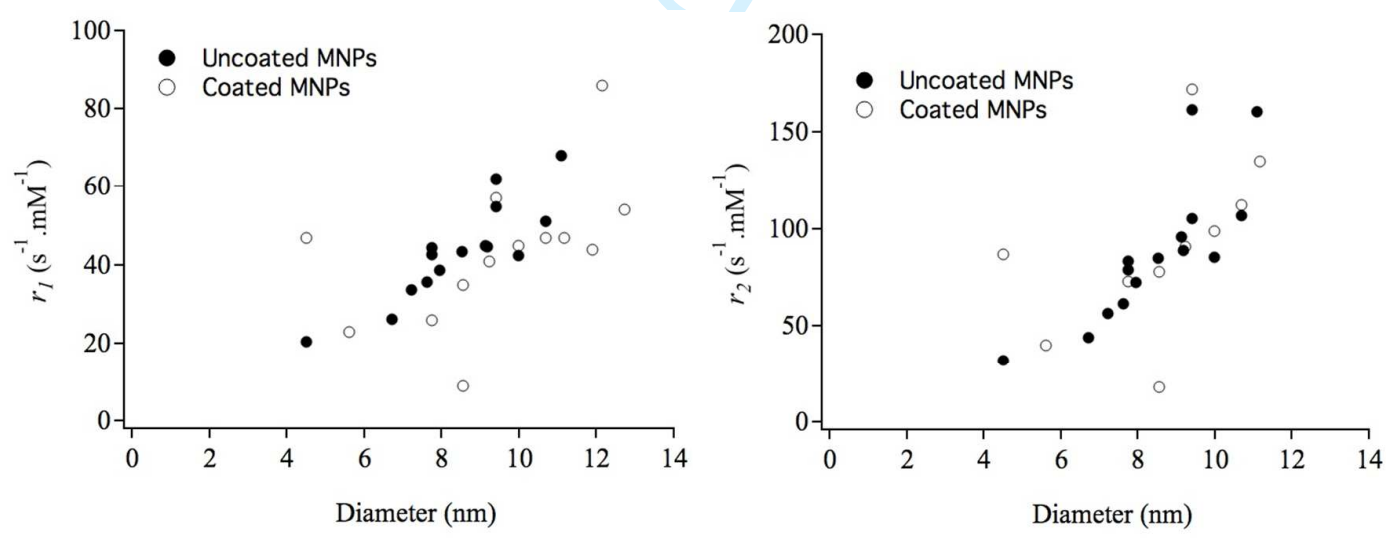

Figure 5: $r_{1}$ and $r_{2}$ relaxivities of uncoated (black symbol) and coated (empty symbol) maghemite as a function of the diameter (for $\sigma \leq 0.2$ ). 
1

2

3

4

5

6

7

8
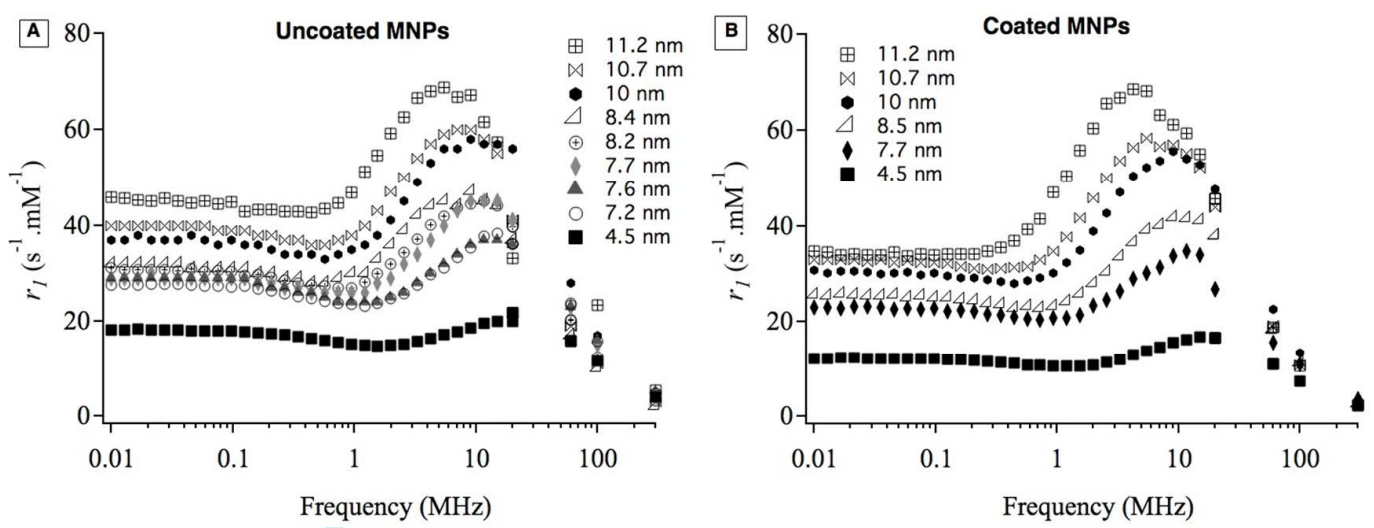

Figure 6: $r_{l}$ profile of maghemite uncoated MNPs (A) and PAAMA-coated MNPs (B) for diameters of the maghemite core (polymer corona not included) ranging from 4.5 to $11.2 \mathrm{~nm}$.

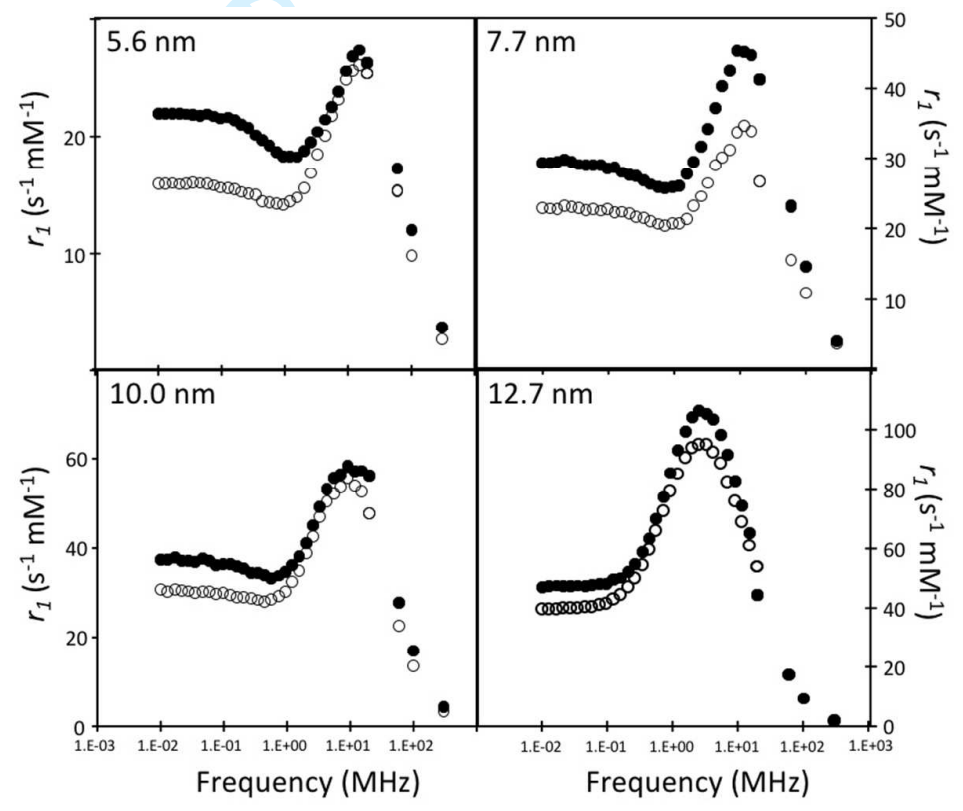

Figure 7: $r_{l}$ profile of maghemite MNPs non coated (black symbol) and PAAMA coated (empty symbol) for several diameters of the maghemite core (polymer corona not included): 5.6, 7.7, 10 and $12.7 \mathrm{~nm}$. 

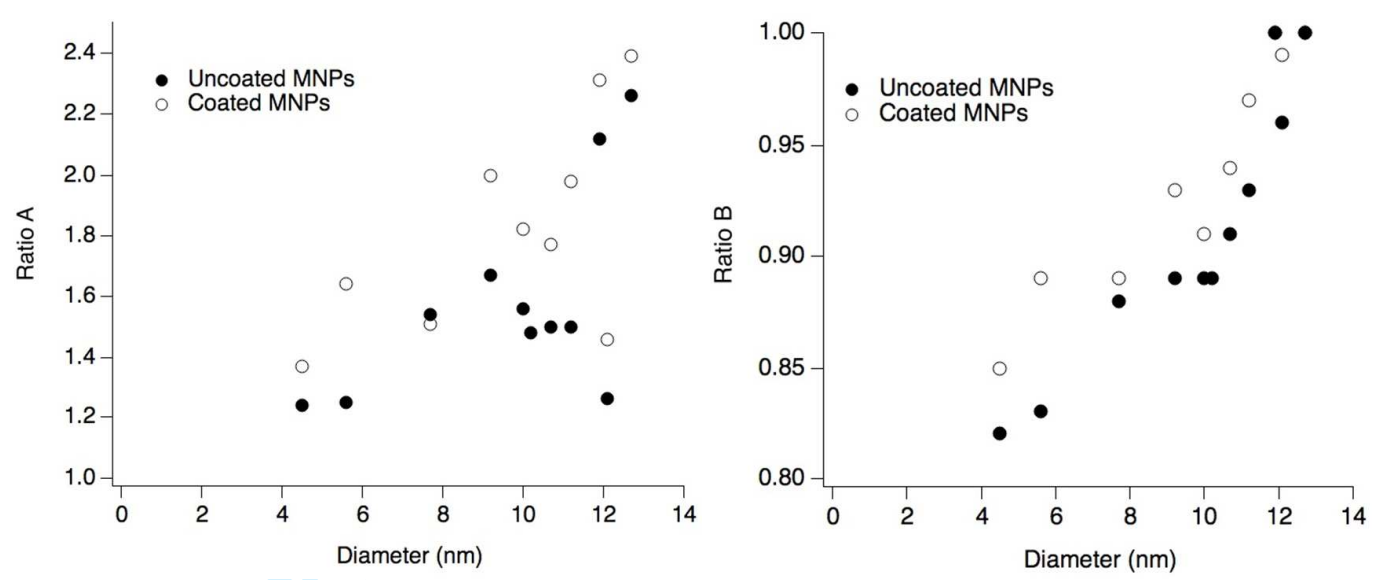

Figure 8: ratio $A$ between the maximum of $r_{l}\left(r_{l}{ }^{\max }\right)$ and the $r_{1}$ at $10 \mathrm{kHz}$ (the plateau value $\left.r_{l}^{\text {plateau }}\right)$ and the ratio $B$ between the minimum of $r_{l}$ before the bump $\left(r_{l}{ }^{\text {deep }}\right)$ and the $r_{l}{ }^{\text {plateau }}$ as a function of the maghemite diameter for uncoated (black disks) and coated (empty circles) MNPs.
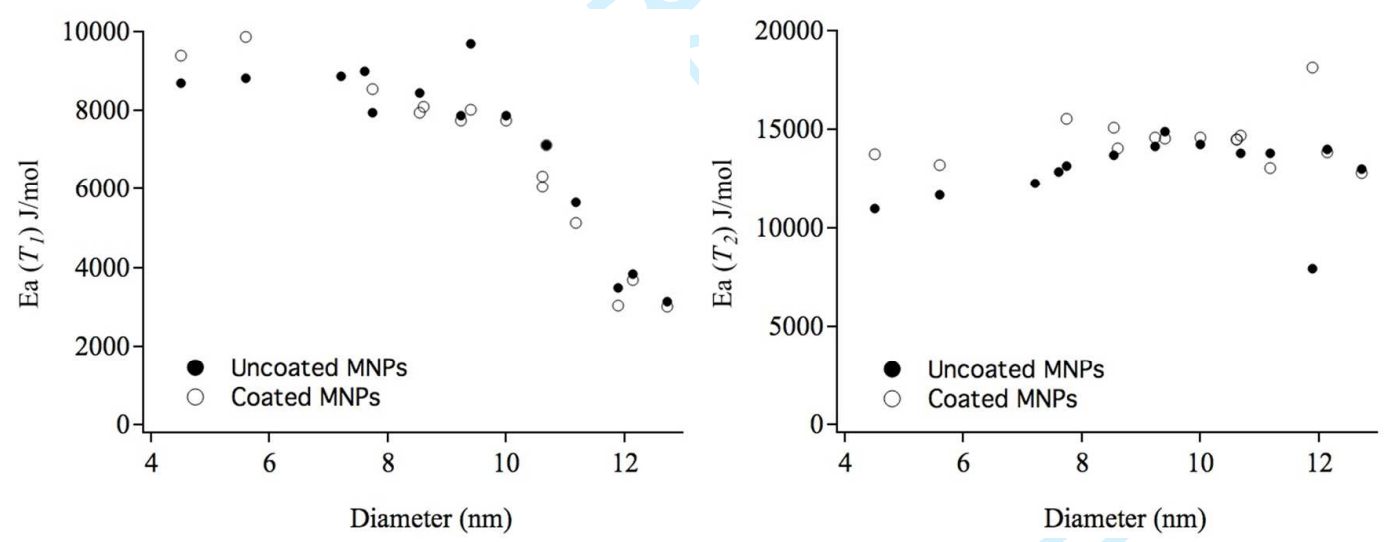

Figure 9: Activation energy Ea of $T_{1}$ (left) and $T_{2}$ (right) Ea for non-coated (black symbol) and coated (empty symbol) maghemite as a function of the diameter. 
1

2

3

4

5

6

7

8

9

10

11

12

13

14

15

16

17

18

19

20

21

22

23

24

25

26

27

28

29

30

31

32

33

34

35

36

37

38

39

40

41

42

43

44

45

46

47

48

49

50

51

52

53

54

55

56

57

58

59

60
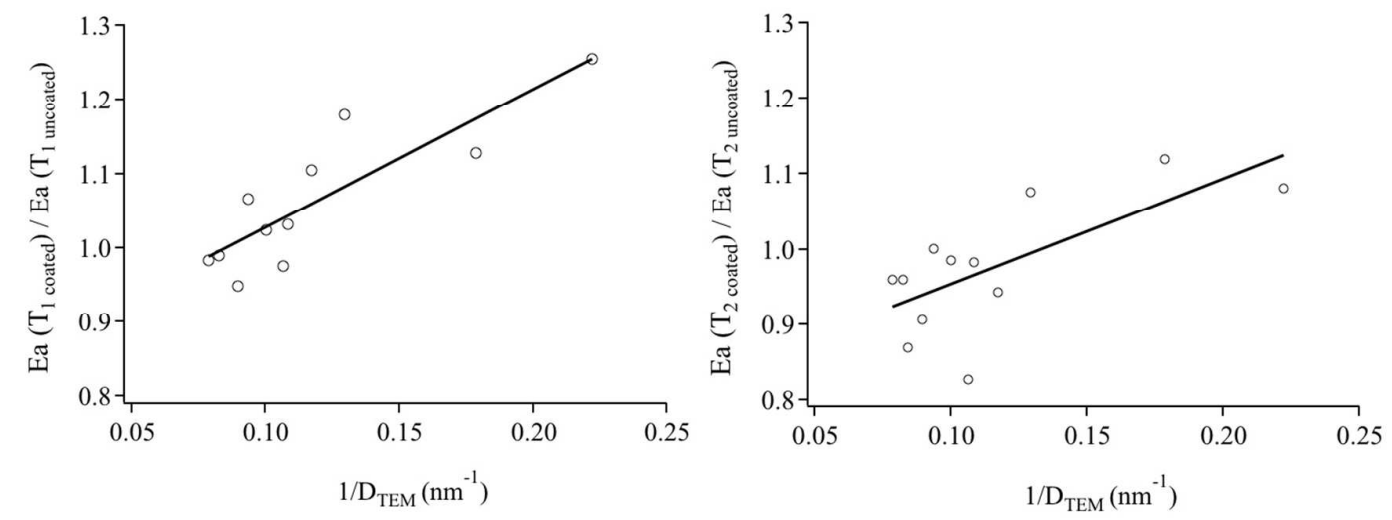

Figure 10: ratio of activation energy Ea of $T_{1}$ (left) and $T_{2}$ (right) Ea between non-coated and coated maghemite as a function of the inverse of the diameter. Lines are linear fits. 